\title{
Designing a Mobile Task Based UI for Tourists
}

\begin{tabular}{|c|c|c|}
\hline Lieve Laporte & Peter Eyckerman & Bieke Zaman \\
\hline er for User Experience Research & Center for User Experience Research & Center for User Experience Research \\
\hline K.U.Leuven & K.U.Leuven & K.U.Leuven \\
\hline Parkstraat 45 Bus 3605 & Parkstraat 45 Bus 3605 & Parkstraat 45 Bus 3605 \\
\hline 3000 Leuven & 3000 Leuven & 3000 Leuven \\
\hline +3216320479 & +3216321869 & +3216323651 \\
\hline lieve.laporte@ & peter.eyckerman@ & bieke.zaman@ \\
\hline soc.kuleuven.be & soc.kuleuven.be & soc.kuleuven.be \\
\hline
\end{tabular}

\begin{abstract}
Substantial research effort has been devoted to the design of mobile tourist guides. Although a lot of these guides already provide some naturalness and intelligence in the interaction with users, their user interfaces are mostly object-based. In this article we will argue that, due to the users' limited attention capacity and the great variety of tasks they have to perform on their trips, more emphasis should be put on the integration of tasks within the users' context of activity. Based on the preliminary results of a qualitative user \& task analysis, we suggest concrete guidelines and a task hierarchy for a mobile tourist guide UI.
\end{abstract}

\section{Categories and Subject Descriptors}

H.5.2 [Information Systems]: User Interfaces - graphical user interfaces, prototyping, user-centered design.

\section{General Terms}

Design, Human Factors

\section{Keywords}

Electronic tourist guides - Task-based interfaces

\section{INTRODUCTION}

In recent years, research on the use of technology in non-work settings has expanded. Particularly mobile devices have proven to be very applicable in these new contexts. One of the most promising application fields for the use of mobile devices in constantly changing environments is tourism. Digital mobile tourist guides, providing geographic and other tourist information, can help users deal with a lot of practical difficulties they are facing when on a trip. Substantial research effort has been devoted to mobile tourist services using Location Based Systems, though with different focus. It is remarkable that most of the prototypes that have been developed, and most mobile interfaces in general for that matter, are organized from an object-based viewpoint [1][2]. Less research has been performed on the potential and design of a full task-based mobile system, although a task-based system is more efficient for information retrieval than a keyword or directory search [3]. In this paper, we will report on the preliminary results of the design of a task-based UI for a mobile tourist guide. We will first explain the difference between both object- as well as task-based interfaces (TBIs). Then, we will report on the preliminary results of a user and task analysis and define a task hierarchy and concrete guidelines for Copyright is held by the author/owner(s).

MobileHCI’09, September 15 - 18, 2009, Bonn, Germany.

ACM 978-1-60558-281-8. the design of mobile tourist guide UIs.

\section{TASK BASED USER INTERFACES 2.1 Comparing task and object-based UIs}

A few definitions of TBIs can be found like [4][5][6]. We conceptualize a TBI simply as an interface where navigation is based on tasks, which allows users to concentrate on what they want to do instead of how to do it. TBIs start with selecting the task at hand, in contrast to object-based UIs that are centered on selecting applications, documents, files or information [7].

E.g. imagine a tourist in Paris wanting to visit the Louvre museum. In an object-based city guide he has to look for a section on culture or museums and find the item for the Louvre to find its location and opening hours (the object). Then he has to look elsewhere for a section on public transport or a map to find out how to get there (the action).

In a TBI the tourist selects "visiting" (the task). Then he selects the object to visit: a museum, the Louvre. The UI then offers him all he may need to perform the task (a description, a map with the route from his current location to the Louvre, the metro line).

The advantage of TBIs over object-based UIs is that the first are less cognitively demanding because they do not require users to translate "what they want to do" to "name of the menu" before realizing a task [8]. Reducing the mobile system's learning curve to a minimum is especially pertinent for tourist situations which are typically complex and unpredictable.

\subsubsection{Method}

Hardly any literature is available on designing TBIs. We will discuss (how we came to) concrete guidelines of how such TBIs should be designed to be optimally adapted to the tourist context of use, causing a minimum of cognitive efforts to the user.

In the process of developing a TBI for a digital mobile guide, we first conducted an exploratory user and task analysis. Our goal was two-fold: we wanted to get a view of (1) users' typical activities (or tasks) while travelling, and (2) the way they make decisions, the information they need to make the decisions, and the way they want to retrieve this information.

A group of users going on a city trip were provided with diaries to report their daily travel activities. After their trip they were interviewed. The diaries were structured in the sense that they offered the user some questions and assignments that were used as a steppingstone during the interview. Apart from some general questions about the users' preferred kind of trips, way of travelling and motivation to travel, the diaries were mainly 
designed to inquire after users' activities and decisions and the information they use to make these decisions while on a trip. For example, users were asked to describe in great detail an activity such as a "museum visit", splitting it up into as many subtasks as possible (e.g. reading about the museum, finding the museum on the map, deciding on transport to the museum, going to the museum, etc.). In addition, with the card sorting method we were able to group similar tasks.

\subsubsection{Preliminary results}

From the diaries and the interviews, complemented with research on TBIs, a list of UI design recommendations was made:

- Provide an easy way to switch between menu and map (e.g. like leafing through a book), as users often use travel guide and map at the same time. For the same reason, points of interest from the menu should also be shown on the map.

- Provide links to secondary or alternative tasks (e.g. offer task "eat and drink" as a subtask of "visit"), as users are very flexible in changing their plans, depending on the context.

- Provide a general task "bookmark” and make this very obvious, as users often discover things on their trip that they want to visit at another moment.

- Use the names of the tasks, as users would like their tasks in real life to map the tasks in their tourist guides.

- Provide consistent screens or parts of screens for tasks that reoccur on different places in the task hierarchy (e.g. the task “find POI").

- Provide explicit screens for the most important starting tasks, as users agree in considerable measure on the 'main travelling activities'.

Further, the activities described by the users, the decisions they made while on a trip and the information they needed for making these decisions, served as input for the development of a visual task hierarchy. Figure 1 presents a small part of the task hierarchy, illustrating a task-way representation of the user's activity. The bold rectangles in this figure represent activities that were agreed upon by users as their 'main travelling activities'. These activities or tasks can be decomposed in subtasks, each of which constitutes a step in achieving the main task. Subtasks are specific to a particular activity (e.g. 'navigate to POI', marked with regular lines in Figure 2), but they can also be common to a lot of activities (e.g. 'find POI', which is common to 'visit' and 'eat and drink'). Besides these subtasks, some tasks can also be complemented by 'aligned' tasks, i.e. tasks that are often started after completion or instead of the original task (e.g. 'find alternative POI'). Decomposition of the user's activities in common and specific subtasks, and aligned tasks, can provide useful information for building a visual task hierarchy, as it better reflects the natural way the user chooses his activities and makes his decisions while on a trip.

\section{RESEARCH STATUS}

Although a more thorough research of the advantages and disadvantages of object-based and task-based guide systems is needed, we already want to stress the potential for designing TBIs, an approach that is still too often underestimated. TBIs are indeed adapted to the specific context of mobile tourism in which users have to cope with limited attention capacity and a great variety of tasks. Based on the preliminary results of a user and task analysis and card sorting, we were able to define some concrete guidelines and a task hierarchy for a mobile guide UI. By the time of the conference, we will be able to show more results such as the complete list of guidelines, a detailed task hierarchy and ontology as well as the first inspiring prototypes.

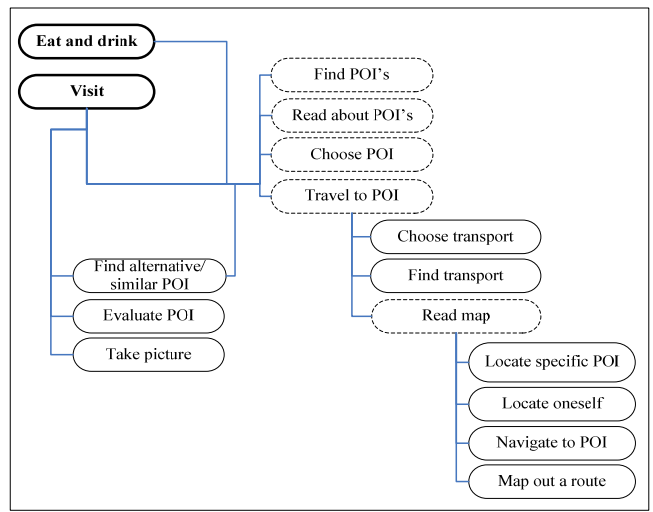

Figure 1. Part of a preliminary task hierarchy for a digital mobile guide user interface.

\section{ACKNOWLEDGMENTS}

Our thanks to the partners in the TALOS project (FP7 Research for SMEs work program of the European Commission).

\section{REFERENCES}

[1] Kenteris, M., Gavals, D. and Economou, D. An innovative mobile electronic tourist guide application. Personal Ubiquitous Computing, 13, 2009, 103-118.

[2] Milic-Frayling, N., Hicks, M., Jones, R. and Costello, J. On the design and evaluation of web augmented mobile applications. Proc. MobileHCI, 2007.

[3] Naganuma, T and Kurakake, S. Task Knowledge Based Retrieval for Services Relevant to Mobile User's Activity. In The Semantic Web - ISWC 2005. Springer, 2005, 959-973.

[4] Zimmerman G. Open User Interface Standards - Towards Coherent, Task-Oriented And Scalabe User Interfaces In Home Environments. Proc. IET IE07, 2007.

[5] Yu, J. et al. Task Based User Interface. US Patent 20080046834, 2008.

[6] Thurrott P. How it works: Inductive user interfaces. Retrieved June 10, 2009: http://www.winsupersite.com/ showcase/longhorn_task-based_ui.asp

[7] Sun Web Application Guidelines - Version 4.0. Retrieved May 7, 2009, from Sun Microsystems: http://developers.sun. com/docs/web-app-guidelines/uispec4_0/05navigation.html\#5.

[8] Sasajima, M., Kitamura, Y., Naganuma, T., Kurakake, S., Mizoguchi, R. Task Ontology-Based Framework for Modeling Users' Activities for Mobile Service Navigation. ESWC Proceedings, 2006. 